\title{
The role of mutations at codons 32, 47, 54, and 90 in $\mathrm{HIV}-1$ protease flap dynamics
}

\author{
Poorvi Chordia ${ }^{1,2, \#, ~ T a m a r i a ~ G . ~ D e w d n e y ~}{ }^{1, \#, \text { Bradley Keusch }}{ }^{1}$, Benjamin D. Kuiper ${ }^{l}$, Kyla \\ Ross $^{1}$, Iulia A. Kovari ${ }^{1}$, Rodger MacArthur ${ }^{2}$, Hossein Salimnia ${ }^{3}$, Ladislau C. Kovari ${ }^{1, *}$
}

${ }^{1}$ Department of Biochemistry and Molecular Biology, ${ }^{2}$ Department of Infectious Diseases, ${ }^{3}$ Department of Pathology, Wayne State University School of Medicine, Detroit, Michigan, USA

\# These authors contributed equally to this work;

*Corresponding author: Ladislau C. Kovari, PhD, Department of Biochemistry and Molecular Biology, Wayne State University School of Medicine, Detroit, Michigan, USA 540 E. Canfield Street, Detroit, MI 48201, USA. E-mail: kovari@med.wayne.edu

Submitted: July 31, 2014; Revised: Dec. 23, 2014; Accepted: Dec. 28, 2014; Published: Dec 31, 2014;

Citation: Chordia P, Dewdney TG, Keusch B, Kuiper BD, Ross K, Kovari IA, MacArthur, Salimnia H, Kovari LC. The role of mutations at codons 32, 47, 54, and 90 in HIV-1 protease flap dynamics. Discoveries 2014, OctDec; 2(4): e27. DOI: 10.15190/d.2014.19

\begin{abstract}
Treatment of Human Immunodeficiency Virus remains challenging due to the emergence of drug resistant strains under the selective pressure produced by standard anti-retroviral therapy. To explore the structural mechanisms of drug resistance, we performed $40 \mathrm{~ns}$ molecular dynamics simulations on three multi-drug resistant HIV-1 protease clinical isolates from patients attending an infectious diseases clinic in Detroit, MI. We identified a novel structural role for the I47V, V32I, I54M and L90M major resistance mutations in flap opening and closure of MDR-PR isolates. Our studies suggest that I47V is involved in the flap opening and the interaction between I47V and V32I tethers the flaps to the active site. I54M and L90M may be responsible for asymmetric movement of the protease flaps. These findings can be utilized to improve drug design strategies against MDR HIV-1 PR variants.
\end{abstract}

\section{Keywords:}

HIV-1 protease, molecular dynamics, drug resistance, protease mutations

\begin{abstract}
Abbreviations:
Human Immunodeficiency Virus (HIV); Highly Active AntiRetroviral Therapy (HAART); Multi-Drug Resistant (MDR); Food and Drug Administration (FDA); Protease (PR); Detroit Multi-Drug Resistant isolates (DetMDR); Protease Inhibitor (PI); Darunavir (DRV); Lopinavir (LPV); Tipranavir (TPV); Atazanavir (ATV); Saquinavir (SAQ); Indinavir (IND); Molecular Dynamics (MD); Root Mean Square Deviation (RMSD); Wild Type (WT); van der Waals (vdW);
\end{abstract}

\section{INTRODUCTION}

The high viral replication rate and error-prone reverse transcriptase leads to the emergence of drug resistant human immunodeficiency virus variants under the selective pressure of anti-retroviral

$\checkmark$ I47V HIV-1 mutation is involved in flap opening and the interaction between I47V and V32I tethers the flaps to the active site

\ These findings are important in drug design strategies 
Table 1. Sequence alignment of WT, MDR-769, DetMDR1-3

\begin{tabular}{|l|llll|}
\hline NL4-3 & PQITLWKRPL & VTIKIGGQLK & EALLDTGADD & TVLEEMNLPG \\
& RWKPKMIGGI & GGFIKVRQYD & QILIEICGHK & AIGTVLVGPT \\
& PVNIIGRNLL & TQIGCTLNF & & \\
\hline MDR-769 & PQITLWKRPI & VTIKIGGQLK & EALLDTGADD & TVLEEVNLPG \\
& RWKPKLIGGI & GGFVKVRQYD & QVPIEICGHK & VIGTVLVGPT \\
& PTNVIGRNLM & TQIGCTLNF & & \\
\hline DetMDR1 & PQITLWKRPV & ITVKIAGQLK & EALLDTGADD & TIFEEMNLPG \\
& RWTPKIVGGI & GGEMKVRQYD & QIPIEICGHK & LVGPVLVGPT \\
& PTNVIGRNMM & TQLGCTLNF & & \\
\hline DetMDR2 & PQITLWKRPV & VTVKVGGQLF & EALLDTGADD & TVFEEINLPG \\
& RWKPKIVGGI & GGFVKVRQYD & QILIEICGKK & IISTVLVGPT \\
& PVNVIGRNTL & TQMGCTLNF & & \\
\hline DetMDR3 & PQITLWKRPF & VTVKIGGQLI & EALLDTGADD & TIFEEMNLPG \\
& RWKPKIIGGI & GGFLKVRQYD & QILIEICGHK & AIGTVVVGPT \\
& PVNVIGRNML & TQIGCTLNF & & \\
\hline
\end{tabular}

therapy ${ }^{1,2}$. The current standard treatment of HIV utilizes HAART where the PIs represent a key class of drugs ${ }^{3}$. PIs have a higher barrier of resistance relative to other classes of inhibitors, as multiple protease mutations are needed for a patient to develop resistance ${ }^{4-6}$. Although there are nine United States FDA approved PIs, some first generation inhibitors (e.g. indinavir, saquinavir, and nelfinavir) are considered obsolete by clinicians due to emergence of drug resistance or from their side effects $^{3}$. Second generation PIs, especially darunavir and tipranavir, remain potent against mutant viral strains that are resistant to first generation $\mathrm{PIs}^{7,8}$. With the availability of second generation PIs, treatment of patients with MDR HIV is possible and can result in full viral suppression ${ }^{9}$. Many factors ${ }^{10-25}$, including mutations in the viral polyprotein (particularly in Gag and Env $)^{21}$, the development of cross-resistance, ${ }^{10}$ and the high mutability of the HIV-PR ${ }^{11}$ continue to produce viral strains that are resistant to second generation PIs ${ }^{12,13}$. Therefore, novel and potent new drugs are required for the treatment of multi-drug resistant HIV infection.

This study utilized molecular dynamics simulation techniques in order to better understand the molecular mechanisms by which MDR-PRs are able to evade inhibition by potent PIs. Clinical MDR-PR isolates were obtained from three patients at the Wayne State University Infectious Disease Clinic in Detroit, MI. On a boosted PI regimen, these patients had a low CD4 cell count and high HIV viral load, indicating treatment failure.
Genotypic and virtual phenotypic analysis of the protease genes showed multiple major and accessory drug resistance mutations which conferred extensive resistance to the nine FDA approved PIs.

The HIV PR is a homodimer containing 99 amino acid residues per monomer ${ }^{14}$. The DetMDRs differ from isolates previously studied by our group in that these contain the major drug resistance mutations L33F, I47V, I50V, I54M, L76V, V82I/F, and $\mathrm{I} 84 \mathrm{~F}$ not present in the previous cohort ${ }^{7}, 16-18$ (Table 1). The DetMDRs also contain previously identified non-polymorphic accessory mutations L10V/G, V11I, I13V, K20T/R, L33F/I/M, K43T, F53L, A71L, T74P, and L89V. To understand the molecular mechanisms of resistance of the MDR HIV PR, 40 ns molecular dynamics simulations of apo and complexed DetMDRs were performed. These studies provide insight into the structural changes that lead to multidrug resistance; in particular, we identify previously unreported roles for the I47V, V32I, I54M, L90M mutations in protease flap dynamics (Figure 1).

\section{MATERIALS AND METHODS}

MDR genotype and virtual phenotype of Detroit isolates

Sequence comparisons of the Detroit isolates highlight the differences as well as the common mutations present in each isolate (Table 1). All three isolates contain the L33F, M46I, and I84V major drug resistance mutations and the $\mathrm{I} 13 \mathrm{~V}$ 


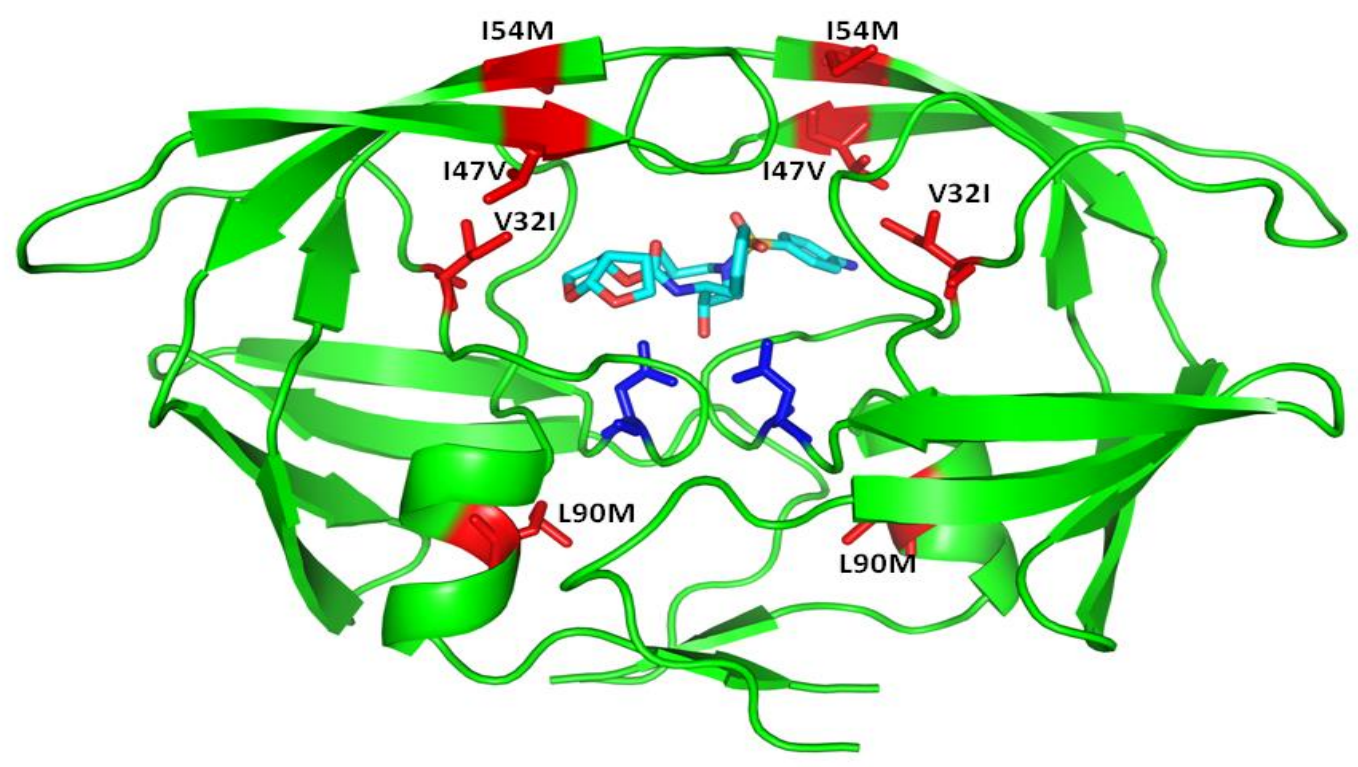

Figure 1. Structure of the HIV-1 PR in complex with the PI darunavir.

The HIV-1 PR is shown as a cartoon in green. The key residue mutations contributing to flap dynamics are shown in red. The catalytic aspartic acid residues are shown in blue. Darunavir is bound to the active site in cyan.

accessory mutation. The protease sequences of the Detroit isolates were submitted for virtual phenotype predictions with vircoTYPE (Table 2). This software uses an input sequence, along with known information regarding resistance mutations to calculate quantitative levels of phenotypic susceptibility to the entire class of PIs 19. Clinicians use this information as a guide when prescribing individualized therapy for their HIV infected patients. The vircoTYPE predictions of the Detroit isolates suggest that DetMDR1 is highly resistant to all nine FDA approved PIs. DetMDR2 is susceptible to DRV with reduced susceptibility to IND, SAQ, LPV, and ATV. DetMDR3 is predicted to be susceptible to SAQ and TPV with reduced susceptibility to LPV, ATV, and IND.

\section{Simulation preparation and molecular dynamics}

The crystal structure of the protease-darunavir complex (PDB: 3D20)24 was used to build a

Table 2. Virtual phenotype predictions of the Detroit Isolates with VircoType suggest multidrug resistant phenotype

\begin{tabular}{|c|c|c|c|c|c|c|c|c|c|}
\hline Isolate & IDV & IDV/r & NFV & SQV/r & FPV/r & LPV/r & $\mathbf{A T V} / \mathbf{r}$ & TPV/r & DRV/r \\
\hline Isolate 1 & $\begin{array}{c}87.3 \\
(\operatorname{minR})\end{array}$ & $\begin{array}{c}87.3 \\
\text { (minR.) }\end{array}$ & $\begin{array}{c}65.2 \\
(\operatorname{minR})\end{array}$ & $\begin{array}{c}49.0 \\
(\operatorname{minR})\end{array}$ & $\begin{array}{c}68.5 \\
(\operatorname{minR})\end{array}$ & $\begin{array}{c}113.2 \\
(\operatorname{minR})\end{array}$ & $\begin{array}{c}79.2 \\
(\operatorname{minR})\end{array}$ & $\begin{array}{c}123.2 \\
(\operatorname{minR})\end{array}$ & $\begin{array}{c}208.2 \\
(\operatorname{minR})\end{array}$ \\
\hline Isolate 2 & $\begin{array}{c}22.5 \\
\text { (redR) }\end{array}$ & $\begin{array}{l}22.5 \\
\text { (redR) }\end{array}$ & $\begin{array}{c}34.1 \\
(\operatorname{minR})\end{array}$ & $\begin{array}{l}17.1 \\
\text { (redR) }\end{array}$ & $\begin{array}{c}31.2 \\
(\operatorname{minR})\end{array}$ & $\begin{array}{l}27.5 \\
\text { (redR) }\end{array}$ & $\begin{array}{c}28.9 \\
(\mathrm{redR})\end{array}$ & $\begin{array}{c}16.1 \\
(\operatorname{minR})\end{array}$ & $\begin{array}{c}9 \\
(\max R)\end{array}$ \\
\hline Isolate 3 & $\begin{array}{c}8.8 \\
(\operatorname{minR})\end{array}$ & $\begin{array}{c}8.8 \\
\text { (redR) }\end{array}$ & $\begin{array}{c}10.1 \\
(\operatorname{minR})\end{array}$ & $\begin{array}{c}0.9 \\
(\operatorname{maxR})\end{array}$ & $\begin{array}{c}70.0 \\
(\operatorname{minR})\end{array}$ & $\begin{array}{c}39.4 \\
\text { (redR) }\end{array}$ & $\begin{array}{c}17.8 \\
\text { (redR) }\end{array}$ & $\begin{array}{c}0.7 \\
(\operatorname{maxR})\end{array}$ & $\begin{array}{c}151.6 \\
(\operatorname{minR})\end{array}$ \\
\hline
\end{tabular}

minR: Minimal Response; redR: Reduced Response; $\max R$ : Maximal Response; $\mathbf{X} / \mathbf{r}=$ ritonavir boosted 


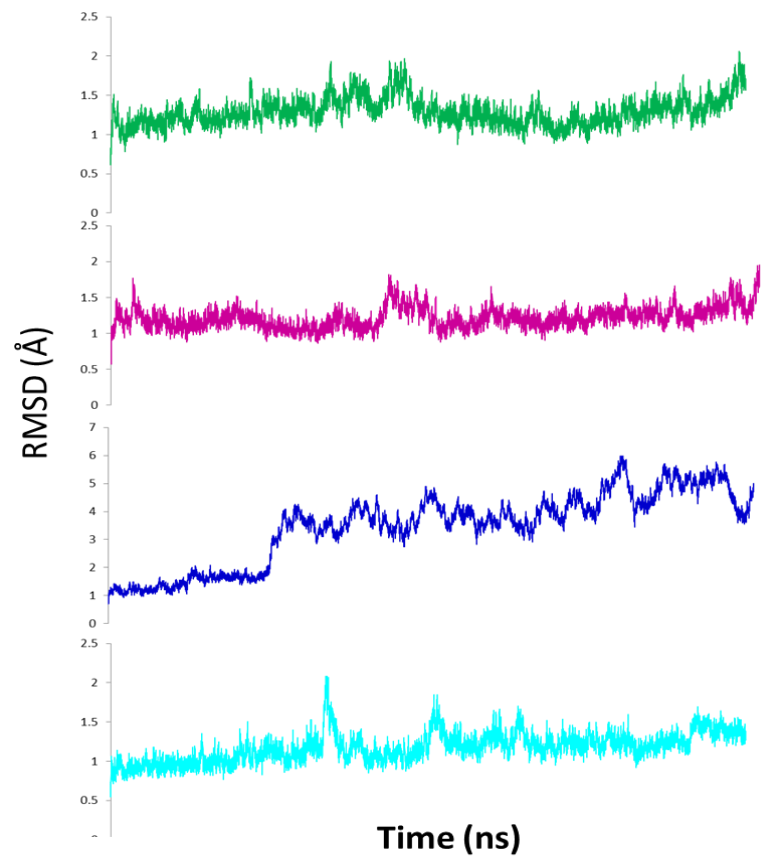

structure based model that include the DetMDR mutations and the NL4-3 wild type template (Table 1). All residues were mutated using the mutagenesis tool in PyMol20. Based on the catalytic mechanism of HIV-1 protease, Asp 25 was assigned a protonated state while Asp 25' was assigned a deprotonated state to prevent ionic repulsion during the simulation. All histidine residues were assigned a neutral charge. Crystallographic water molecules were retained in the initial setup and the ligand was removed for the apo simulations. The coordinates for the ligands DRV, ATV, and LPV were obtained from structures in the PDB (3D20, 3OXX, and $2 \mathrm{O} 4 \mathrm{~S})^{24,25}$.

The MD simulations were performed using the parallel computing program Scaling NAno Molecular Dynamics (NAMD) V. 2.9 23 . The PR complex models were solvated in a $12 \AA$ water box using TIP3P models for water molecules ${ }^{22}$. The cutoff for non-bonded interactions was $10 \AA$ A. The Particle Mesh Ewald method was implemented to calculate long-range electrostatic interactions. The systems were energy minimized using 20,000 steps of the conjugate gradient method and gradually heated from $0 \mathrm{~K}$ to $300 \mathrm{~K}$. Simulations were conducted in the isobaric-isothermal ensemble at $300 \mathrm{~K}$ and $1.0 \mathrm{~atm}$ (NPT ensemble) for $40 \mathrm{~ns}$ using the CHARMM all-atom force field 36 and a time step of 2 fs. Langevin dynamics ensured a constant temperature, with a damping coefficient of 5 ps-1.
Figure 2. Root mean square deviation (RMSD) of Ca positions of the apo HIV-1 protease isolates.

Wild-type is shown in green, DetMDR1 in pink, DetMDR2 in blue and DetMDR3 in cyan.

Note: DetMDR2 plot (blue) has altered yaxis scale.
The Nose-Hoover piston method maintained a constant pressure of $1 \mathrm{~atm}$.

Data analysis of molecular dynamics simulation Trajectories of MD simulation were visualized and analyzed using the Visual Molecular Dynamics (VMD) program v 1.91. The RMSD values were calculated using the VMD RMSD trajectory plug-in. The hydrogen bonding network was calculated using the $\mathrm{H}$-bond tool in VMD, with $3.0 \AA$ distance and 20 degree angle cutoffs. Structure figures were made using PyMol.

\section{RESULTS}

Mutations in the Detroit isolates reveal alternative protein dynamics

RMSD analysis of the apo structure of the DetMDR isolates show increased fluctuation of all three isolates compared to the WT PR (Figure 2). DetMDR1 shows only a brief increase in flexibility while DetMDR2 shows a sharp increase followed by sustained elevation in RMSD for the rest of the trajectory. DetMDR3 displays short bursts of increased flexibility but no sustained changes in RMSD. These data suggest a large conformational change in DetMDR1 and DetMDR2 but not in DetMDR3 or the WT. 


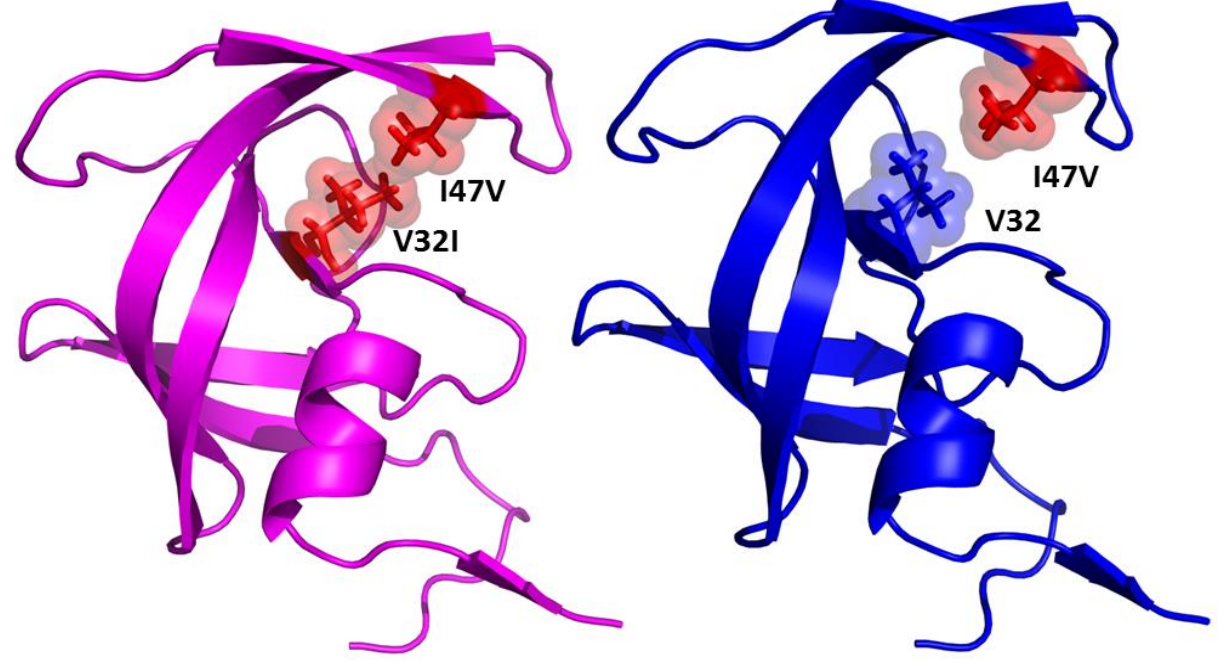

Figure 3: Change in the van der Waals volume induced by the drug resistance mutations I47V and V32I. The left panel shows DetMDR1; the right panel shows DetMDR2.

V32I interacts with I47V to tether the protease flaps in a closed conformation

Visual analysis of the trajectory shows that these increases in RMSD are due to opening of the flaps in DetMDR1 and DetMDR2. In DetMDR1 the flaps open and then close. In contrast, the flaps of DetMDR2 open and stay open for the remainder of the trajectory. The WT and DetMDR3 flaps do not open during these simulations. These differences in flap movement correspond to the presence or absence of particular mutations. I47V likely contributes to flap opening as it is present in both isolates 1 and 2 where the flaps open but not in DetMDR3 where the flaps do not open. However, $\mathrm{vdW}$ interactions between the V32I and I47V mutations cause flap closure in HIV-1 PR (Figure 3). These vdW interactions could explain the flap opening followed by quick flap closure observed in DetMDR1, while the absence of V32I in DetMDR2 removes this interaction and cause the flaps to remain open. Similarly, the WT and DetMDR3 have V32 and I47 at these loci and therefore the vdW interactions in the WT and DetMDR3 are maintained casing the flaps to remain closed.

I54M in combination with L90M plays a role in asymmetric flap movement

I54M and L90M are associated with asymmetric movement in DetMDR1 corresponding to opening of the flaps. The RMSD of L90M on chain B is different than that of chain A. In DetMDR1 the asymmetric movement of the flaps as indicated by the differences in RMSD at each residue show a difference in residues 47-54 on chain B compared to chain A (Figure 4). Movement of the residues in this region was symmetric in DetMDR2 where the residue fluctuation per residue is conserved between both chains of the PR.

Darunavir, atazanavir and lopinavir binding stabilize the HIV-1 protease flaps

The opening of the flaps observed in the apo simulations of DetMDR1 and DetMDR2 did not occur in the drug complex simulations. Drug binding maintains a closed flap conformation in DetMDR2. This suggests that the structural mechanism of drug resistance in these isolates is not due to changes in protein flexibility (Figure 5).

\section{Mutation induced changes to the hydrogen bonding} network alters inhibitor conformation

In addition to the altered protein dynamics, there is a change in the hydrogen bonding interactions in the WT compared to the MDR complex (data not shown). DetMDR1 has 2.3\%, $0.8 \%$ and $19.2 \%$ reduced hydrogen bond formation (compared to the WT) when complexed with DRV, ATV, and LPV respectively. Interestingly, DetMDR2 and DetMDR3 have increased hydrogen bond formation when in complex with DRV and ATV and a 21\% 


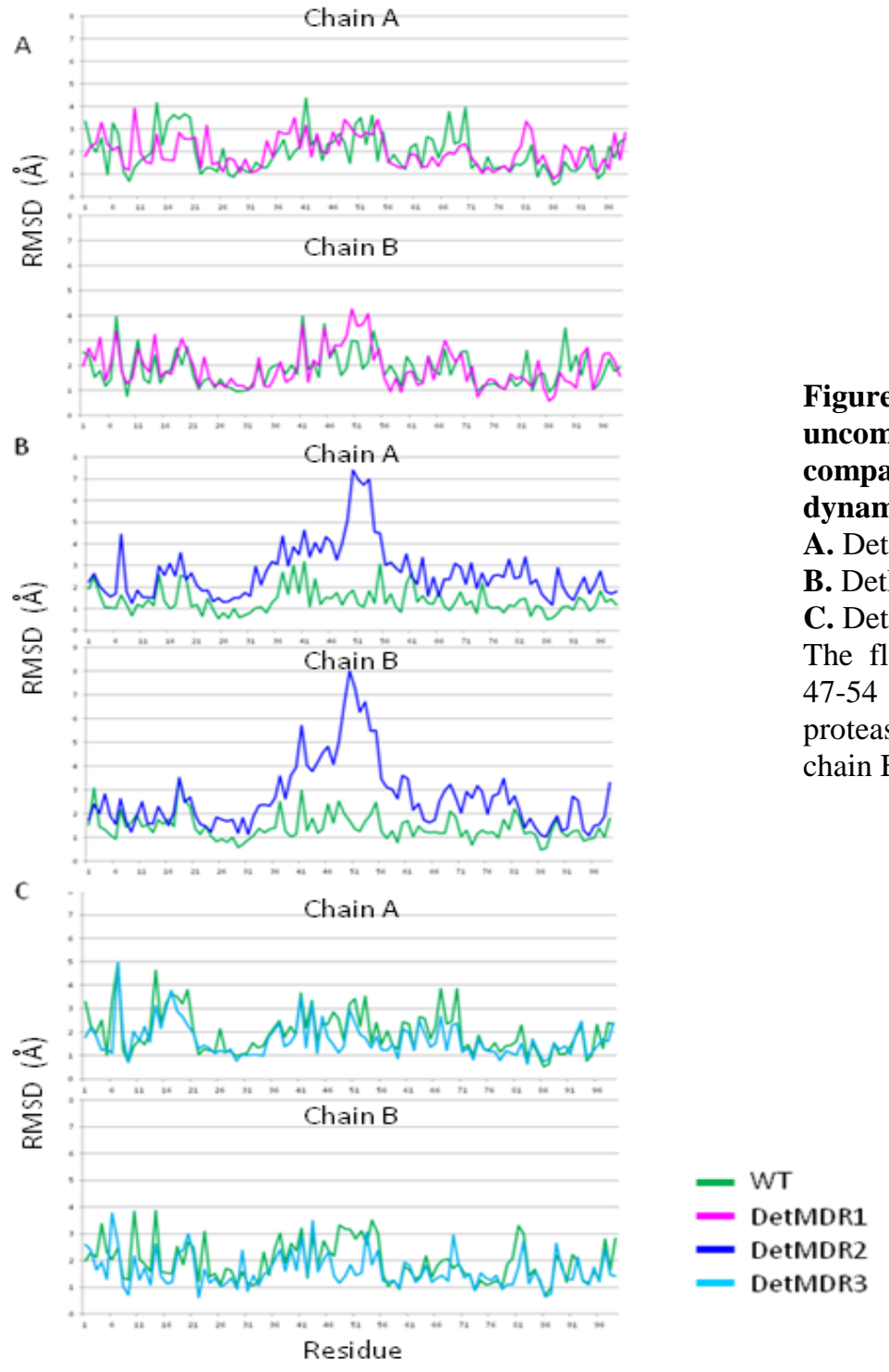

and $36 \%$ decrease in hydrogen bond formation when in complex with LPV. The increase in hydrogen bonding is likely due to an alteration in the residues involved in an interaction with the PI. While changing the binding pocket may increase hydrogen bond formation, this also alters the conformation of the inhibitor.

\section{CONCLUSION}

Three multi-drug resistant HIV-1 protease clinical isolates were selected from patients attending the Wayne State University infectious diseases clinic and failing antiviral treatment therapy on PI based regimens. To explore the structural mechanisms resulting in treatment failure, we performed 40ns MD simulations on the Detroit MDR series. Our results indicate a novel structural role for the I47V, V32I, I54M and L90M resistance mutations.

The V32I and I47V mutations play a structural role in tethering the flaps to the active site. Sequence analysis comparisons of the DetMDR protease isolates showed that DetMDR2 does not contain the V32I and I47V mutation combination. It contains only the I47V mutation and without the V32I mutation there is a loss in $\mathrm{vdW}$ contact volume between these two residues. Analysis of this apo PR trajectory displayed a marked increase in RMSD when compared to the WT, and a visual inspection of the trajectory as well 

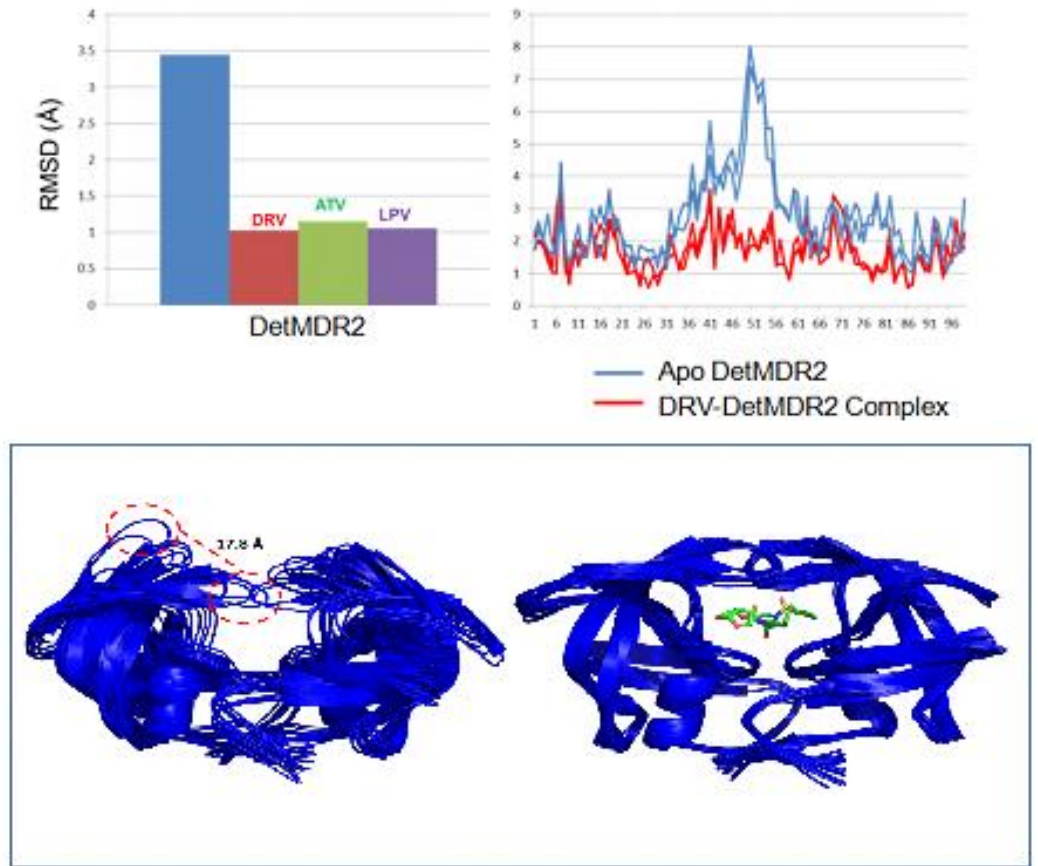

Figure 5: Darunavir, atazanavir and lopinavir binding stabilize the HIV-1 protease flaps.

The top left panel shows the average RMSD of DetMDR2 either alone (blue) or in complex with DRV (red) ATV (green) and LPV (purple). The RMSD per residue is shown in the top right panel. The bottom left panel is representative of the 40ns MD simulation using frames post energy minimization and at 4 ns intervals of uncomplexed and DRV complexed DetMDR2, respectively aligned by the $\mathrm{C} \alpha$ positions. The red dashed lines indicate a $17.8 \AA$ movement of the $\mathrm{C} \alpha$ of Ile50 of the protease flap. This figure was made in PyMol. as per-residue RMSD analysis confirmed that this is due to the flaps opening. Therefore, we postulate that $\mathrm{I} 47 \mathrm{~V}$ is responsible for flap opening. However, V32I is a compensatory mutation that may be responsible for tethering the flaps to the active site through its contacts with I47V. Therefore, if I47V is the only mutation it may be responsible for promoting flap opening through the disruption of the vdW interactions between residues 32 and 47 .

I54M and L90M may be responsible for asymmetric movement of the protease flaps. This mutation combination is only present in DetMDR1 which is the only protease in the series in where the flaps asymmetrically open and then close after 4 ns. The mechanism explaining asymmetric flap opening will be explored further in future characterization studies of the HIV-1 protease isolates.

The role of these structural changes in drug resistance was investigated with the Detroit isolates in complex with the protease inhibitors ATV, DRV, and LPV. These results suggest that drug resistance occurs through alternate hydrogen bonding interactions in each of the mutants. Both the alternate hydrogen bonding networks and the flexibility of the flaps are stabilized with the protease inhibitors bound to the active site.

In summary, this work addresses the role of mutations at codons $32,47,54$, and 90 in HIV-1 protease. We have demonstrated the role of these mutations in the altered flap dynamics. Finally, the reported findings can be utilized to enhance drug design strategies against MDR HIV-1 PR variants.

\section{Acknowledgments}

We thank the National Institutes of Health for funding to LCK (AI65294) and IMSD/MBRS fellowship support to TGD.

\section{Conflict of Interest Statement}

The authors report no conflict of interest.

\section{References}

1. Wainberg MA, Gu Z, Gao Q, Arts E, Geleziunas R, Bour $\mathrm{S}$, et al. Clinical correlates and molecular basis of HIV drug resistance. J Acquir Immune Defic Syndr. 1993;6 Suppl 1:S36-46.

2. Smyth RP, Davenport MP, Mak J. The origin of genetic diversity in HIV-1. Virus Res. 2012 ;169(2):415-29.

3. Tang MW, Shafer RW. HIV-1 antiretroviral resistance: scientific principles and clinical applications. Drugs. 2012;72(9):e1-25.

4. Van Maarseveen N, Boucher C. Resistance to protease inhibitors. In: Geretti AM, editor. Antiretrovir Resist Clin Pract [Internet]. London: Mediscript; 2006 [cited 2013 Nov 7]. Available from: http://www.ncbi.nlm.nih.gov/books/NBK2250/

5. McCoy C. Darunavir: a nonpeptidic antiretroviral protease inhibitor. Clin Ther. 2007;29(8):1559-76. 
6. Johnson VA, Calvez V, Gunthard HF, Paredes R, Pillay D, Shafer RW, et al. Update of the drug resistance mutations in HIV-1: March 2013. Top Antivir Med. 2013; 21(1):6-14.

7. Wang Y, Liu Z, Brunzelle JS, Kovari IA, Dewdney TG, Reiter SJ, et al. The higher barrier of darunavir and tipranavir resistance for HIV-1 protease. Biochem Biophys Res Commun. 2011; 412(4):73742.

8. Berhan A, Berhan Y. Virologic response to tipranavir-ritonavir or darunavir-ritonavir based regimens in antiretroviral therapy experienced HIV1 patients: a meta-analysis and meta-regression of randomized controlled clinical trials. PloS One. 2013;8(4):e60814.

9. Harris M, Nosyk B, Harrigan R, Lima VD, Cohen C, Montaner J. Cost-Effectiveness of Antiretroviral Therapy for Multidrug-Resistant HIV: Past, Present, and Future. AIDS Res Treat. 2012;2012:595762.

10. Rhee S-Y, Taylor J, Fessel WJ, Kaufman D, Towner $\mathrm{W}$, Troia $\mathrm{P}$, et al. HIV-1 protease mutations and protease inhibitor cross-resistance. Antimicrob Agents Chemother. 2010; 54(10):4253-61.

11. Ceccherini-Silberstein F, Erba F, Gago F, Bertoli A, Forbici F, Bellocchi MC, et al. Identification of the minimal conserved structure of HIV-1 protease in the presence and absence of drug pressure. AIDS Lond Engl. 2004; 18(12):F11-19.

12. Spagnuolo V, Gianotti N, Seminari E, Galli L, Fusetti G, Salpietro S, et al. Changes in darunavir/r resistance score after previous failure to tipranavir/r in HIV-1-infected multidrug-resistant patients. J Acquir Immune Defic Syndr 1999. 2009; 50(2):192-5.

13. Lambert-Niclot S, Flandre P, Canestri A, Peytavin G, Blanc C, Agher R, et al. Factors associated with the selection of mutations conferring resistance to protease inhibitors (PIs) in PI-experienced patients displaying treatment failure on darunavir. Antimicrob Agents Chemother. 2008; 52(2):491-6.

14. Wlodawer A, Erickson JW. Structure-based inhibitors of HIV-1 protease. Annu Rev Biochem. 1993; 62:543-85.

15. Wlodawer A. Rational approach to AIDS drug design through structural biology. Annu Rev Med. 2002; 53:595-614.

16. Liu Z, Yedidi RS, Wang Y, Dewdney TG, Reiter SJ, Brunzelle JS, et al. Crystallographic study of multidrug resistant HIV-1 protease lopinavir complex: mechanism of drug recognition and resistance. Biochem Biophys Res Commun. 2013; 437(2):199204.
17. Liu Z, Wang Y, Brunzelle J, Kovari IA, Kovari LC. Nine crystal structures determine the substrate envelope of the MDR HIV-1 protease. Protein J. 2011; 30(3):173-83.

18. Yedidi RS, Proteasa G, Martinez JL, Vickrey JF, Martin PD, Wawrzak Z, et al. Contribution of the 80 s loop of HIV-1 protease to the multidrugresistance mechanism: crystallographic study of MDR769 HIV-1 protease variants. Acta Crystallogr D Biol Crystallogr. 2011;67(Pt 6): 524-32.

19. Van Houtte M, Picchio G, Van Der Borght K, Pattery T, Lecocq P, Bacheler LT. A comparison of HIV-1 drug susceptibility as provided by conventional phenotyping and by a phenotype prediction tool based on viral genotype. J Med Virol. 2009; 81(10):1702-9.

20. The PyMOL Molecular Graphics System, Version 1.5.0.4 Schrödinger, LLC.

21. Rabi SA, Laird GM, Durand CM, Laskey S, Shan L, Bailey JR, et al. Multi-step inhibition explains HIV1 protease inhibitor pharmacodynamics and resistance. J Clin Invest. 2013; 123(9): 3848-3860.

22. Jorgensen WL, Chandrasekhar J, Madura JD, Impey RW, Klein ML. Comparison of simple potential functions for simulating liquid water. J Chem Phys 1983; 79: 926.

23. Phillips JC, Braun R, Wang W, Gumbart J, Tajkhorshid E, Villa E, et al. Scalable molecular dynamics with NAMD. J Comput Chem. 2005; 26(16): 1781-1802.

24. Liu F, Kovalevsky AY, Tie Y, Ghosh AK, Harrison RW, Weber IT. Effect of flap mutations on structure of HIV-1 protease and inhibition by saquinavir and darunavir. J Mol Biol. 2008; 381: 102-115.

25. Muzammil S, Armstrong AA, Kang LW, Jakalian A, Bonneau PR, Schmeimer $\mathrm{V}$ et al. Unique thermodynamic response of tipranavir to human immunodeficiency virus type 1 protease drug resistance mutations. J Virol. 2007; 81: 5144-5154.

DISCOVERIES is a peer-reviewed, open access, online, multidisciplinary and integrative journal, publishing high impact and innovative manuscripts from all areas related to MEDICINE, BIOLOGY and CHEMISTRY; C 2014, Applied Systems 